\title{
Succinic semialdehyde dehydrogenase deficiency
}

INSERM

\section{Source}

INSERM. (1999). Orphanet: an online rare disease and orphan drug data base. Succinic semialdehyde dehydrogenase deficiency. ORPHA:22

Succinic semialdehyde dehydrogenase (SSADH) deficiency is a rare neurometabolic disorder of gamma-aminobutyric acid (GABA) metabolism with a nonspecific clinical presentation (ranging from mild to severe) with the most frequent symptoms being cognitive impairment with prominent deficit in expressive language, hypotonia, ataxia, epilepsy, and behavioral dysregulation. 